\title{
Conectar, calcular, juzgar. D el análisis de redes como fenómeno sociológico
}

Javier Izquierdo

UN ED. Facultad de Ciencias Políticas y Sociología

O bispo Trejo, s/n. 28040 M adrid

\section{Resumen}

El alto grado de penetración alcanzado por las técnicas de cálculo de redes sociales en las prácticas de gestión de las grandes corporaciones empresariales de la «N ueva Economía», ha tenido como consecuencia política más notable el que las convenciones y los aparatos de prueba científico-legal clásicos, orientados a atribuir la autoría y la responsabilidad de ciertos actos a sujetos individuales o corporativos bien delimitados, hayan dejado de constituir referentes naturales para la formación del juicio.

Palabras clave: redes sociales, sociología económica, sentido común de la justicia.

Abstract. Connect,calculate, judge. On the web analysis as a sociological phenomenon

The most important political consequence of the high degree of penetration attained by the al gorithmic techniques for the calculus of «social networks» has been to question the classical legal apparatus of authority and responsibility attribution on a «personal» basis.

Key words: social networks, economic sociology, common sense of justice.

\section{Sumario}

Introducción 3. ¿iH acia un derecho civil de las redes sociales? El ejemplo de la jurisprudencia

1. La ciencia social en una sociedad desencantada (I): el análisis económico de los mercados de red

2. La ciencia social en una sociedad desencantada (II): el análisis de redes sociales estadounidense sobre competencia ilícita en los mercados de red

4. Azar, persona y responsabilidad: un triángulo complicado

Conclusión

Bibliografía 


\section{Introducción}

Una de las corrientes especializadas de más amplio desarrollo académico y éxito mundano del programa general de investigaciones sociológicas de carácter cuantitativo sobre la morfología y densidad de las redes sociales es la llamada «sociología de los mercados» (Swedberg y G ranovetter, 1992). La variable analítica emblemática de esta nueva sociología económica es el capital social, expresado usual mente como una estimación estadística de la «centralidad relativa» de la posición que ocupan diferentes individuos o empresas en una estructura topológica multidimensional, un mercado considerado como una «red social» (W hite, 1980; G ranovetter, 1985 y 1992; Burt, 1988; Baker, 1984 y 1990). En un libro reciente, el profesor Wayne E. Baker, catedrático de conducta organizacional y gestión de recursos humanos en la Escuela de N egocios de la U niversidad de M ichigan y uno de los académicos más prestigiosos en el campo de la investigación cuantitativa sobre los patrones estructurales de formación de capital social, ${ }^{1}$ ha confesado que, a lo largo de sus numerosas charlas y seminarios sobre técnicas de análisis de redes sociales y estrategias de acumulación de capital social, impartidas a una amplia variedad de audiencias académicas y empresariales, ha descubierto que el carácter éticamente cuestionable del «management de relaciones» es una de las preocupaciones que con mayor frecuencia asalta a sus oyentes (Baker, 2001: 19; véase también Scheler, 2002). Baker expone el caso de una de las alumnas de sus seminarios, ejecutiva de una empresa, a la que, tras un diagnóstico del perfil tipo de su red social en el que se destacaba el exiguo tamaño de su núcleo central de relaciones y la extrema similaridad de sus componentes, propuso desarrollar una estrategia orientada a alcanzar un mayor grado de diversidad en sus contactos personales. Para ello, el consultor le recomendaba incluir nuevas personas en su red, y a la vez suprimir algunos de los vínculos existentes. La directiva desechó airadamente el consejo con el siguiente argumento: «N o me gusta pensar que puedo añadir o quitar personas de mi red. No es ético tratar a las personas como cosas que uno puede añadir y sustraer» (ídem 20). La acusación de inmoralidad, oportunismo o sordidez que suele formularse contra quienes se aplican de forma explícita a configurar una cartera óptimamente diversificada de relaciones sociales, es la puerta de entrada de la investigación social al fundamental terreno de la filosofía política - el reino de la justicia. D e esta apertura del paradigma de las redes sociales se ocupa este trabajo.

1. Baker, que obtuvo su doctorado por la Universidad de $\mathrm{N}$ orthwestern a principios de la década de 1980 con una tesis sobre la morfología de las redes sociales de los traders que operaban en el mercado de futuros de C hicago, ha sido durante muchos años profesor asociado en el D epartamento de Sociología de la U niversidad de Chicago (Convert y H eilbron, 1999: 11). En la actualidad ocupa la cátedra de conducta organizacional y gestión de recursos humanos en la Escuela de N egocios de la Universidad de M ichigan. 


\section{La ciencia social en una sociedad desencantada (I): el análisis económico de los mercados de red}

D ado el carácter de «avalancha» (todo o nada) característico de la competencia entre «tecnologías de red», como los aparatos de vídeo o los ordenadores personales, ensamblajes de productos complementarios de software y hardware cuya utilidad para el usuario individual depende de manera crucial del número de usuarios ya existentes, la batalla por «tomar la delantera» obteniendo ligeras ventajas iniciales, bien en términos de conocimiento por los usuarios, bien en forma de base física efectivamente instalada es, junto con la estrategia de $I+D$, el factor determinante de la estructura social de nuestros modernos mercados de información y entretenimiento.

La aceptación de la existencia de mecanismos de retroalimentación positiva en el proceso de coordinación entre los consumidores y de la preeminencia causal que posee la ocurrencia de accidentes o errores al eatorios en los momentos iniciales de una dinámica de competencia económica, ha permitido a la microeconomía contemporánea explicar el carácter estable a largo plazo de equilibrios económicos «subóptimos» en la forma de un proceso de «atrapamiento histórico» (lock-in). El cuerpo de evidencia más conocido - y dis putado (Liebowitz y M argolis, 1990) - en favor de la teoría económica del atrapamiento histórico accidental de estándares tecnológicos subóptimos en mercados de red es un estudio de caso sobre la historia del teclado de las máquinas de escribir. La historia del teclado QWERTY (D avid, 1986) ha sido el germen de una insurgente microeconomía de la organización industrial centrada en el problema de las externalidades de red y la competencia entre sistemas y estándares tecnológicos (Katz y Shapiro, 1994).

En general, desde el trabajo clásico de Ronald Coase (1960) sobre el problema del coste social, el análisis económico de los derechos de propiedad define como «efectos externos» 0 «externalidades» aquellos fenómenos de asignación y redistribución de la riqueza económica que, como consecuencia de la existencia de «costos de transacción» (v. g. los costos en tiempo y recursos de llegar a un acuerdo entre las partes) demasiado altos, no pueden ser capturados dentro del sistema de contratos legales y el mecanismo de formación de precios. Este análisis distingue a su vez entre externalidades negativas (costos económicos soportados por personas no implicadas en su generación) y externalidades positivas (beneficios económicos apropiados por personas no implicadas en su generación). El ejemplo típico de externalidades económicas positivas es la difusión de las innovaciones tecnológicas. Para las externalidades negativas el ejemplo clásico es el del deterioro medioambiental producido por los procesos organizados de producción industrial. D esde este mismo punto de vista, los sistemas legales de registro y protección de patentes y las modernas leyes de protección medioambiental con su aparato de sanciones económicas («quien contamina paga») son, respectivamente, invenciones sociales destinadas a «internalizar» en parte estos dos tipos de externalidades, esto es, a hacer que quienes contaminan y quienes «copian» ilegalmente soporten de algún 
modo los costes sociales implicados en la limpieza medioambiental (mediante el pago de multas) y los costes privados implicados en el I+D empresarial (mediante el pago de cánones o regalías).

Los economistas denominan «mercados de red» (network markets) a aquellos mercados en los que los consumidores desean comprar productos compatibles con aquéllos que compran los demás. Al determinar en parte las estrategias competitivas perseguidas por las empresas, la complementariedad entre productos y la interconexión entre consumidores hace que los mercados de red posean un comportamiento singular. En primer lugar, el juego de la compe tencia en estos mercados suele arrojar resultados del tipo «el ganador se lo lleva todo» (winner takes all): la coexistencia pacífica entre productos incompatibles suele ser inestable y resolverse con un estándar ganador único que «barre» el mercado. En segundo lugar, en los mercados de red no es sólo el volumen actual de ventas o el fondo de ventas acumuladas el factor que permite determinar quien será el ganador. El factor crucial son más bien las expectativas de los consumidores sobre el tamaño final que alcanzará una red.

Los compradores que se unen a lo que acabará siendo la red perdedora debe rán o bien cambiarse, que puede ser muy costoso, o bien contentarse a sí mismos con unos beneficios menores en términos de «externalidades de red» (network externalities) positivas que los que disfrutan los que se unieron a la red ganadora. D ado que las decisiones de los compradores están en este caso fuertemente influidas por sus predicciones de las ventas futuras, son grandes las recompensas que se derivan del trabajo orientado a influir sobre esas expectativas. En estas circunstancias, la victoria empresarial no cae necesariamente del lado del mejor producto o del más barato: un producto inferior puede ser capaz de derrotar a uno superior si existe una expectativa generalizada de que así sea.

Una última característica de los mercados de red es que «la historia importa» (D avid, 1986). M ientras que en otros tipos de mercados los resultados de asignación finales pueden en general ser explicados independientemente de la trayectoria anterior del sistema por el estado actual de las preferencias de los consumidores y las tecnologías de producción, los equilibrios en los mercados de red no pueden entenderse sin conocer el patrón secuencial de decisiones de adopción tecnológica que tuvieron lugar a lo largo de períodos anteriores. Según esta lógica de funcionamiento propiamente «histérica», y considerando que los compradores prefieren disfrutar del máximo grado de compatibilidad con la base de productos ya instalada, sucede en muchos casos que productos «mejores» que arriban al mercado más tarde son incapaces de desplazar a estándares «peores» pero más madrugadores.

El mejor exponente del estado actual de las aplicaciones de este cuerpo de conocimientos expertos en el mundo de la gestión empresarial es un libro publicado originalmente en 1999, El dominio de la información: U na guía etratégica para la economía de la red, obra de dos catedráticos de microeconomía aplicada de la U niversidad de Berkeley (Varian y Shapiro, 2000). La obra establece una serie de conexiones novedosas entre los principios teóricos del análisis económico de la información y la abundante casuística disponible sobre las 
estrategias de gestión de las empresas «punto.com»: diferenciación de precios y calidades, atrapamiento de proveedores mediante contratos de exclusividad, promoción de estándares y defensa agresiva de los derechos de propiedad intelectual (patentes y derechos de autor), aprovechamiento de economías de escala y complementariedades entre productos (soporte / contenido), fidelización mediante descuentos u ofertas de lanzamiento, subvención de costes a los clientes con más visión de futuro o mejor «posicionados» - un teórico de redes diría «con un índice de centralidad más alto»- en el mercado, manipulación de la duración del ciclo de vida de los productos y de los costes de búsqueda de los consumidores, etc. Si bien, como reconocen los autores, en real idad «ninguna de estas estrategias [de gestión empresarial] es completamente nueva» lo cierto es que al ser especialmente aptas para la explotación de productos con ele vados costes fijos de desarrollo y amplios márgenes de beneficio, son «especificamente adecuadas» para el caso de los productos y servicios altamente ricos en información (Varian y Shapiro, 2000: 147).

En el capítulo 6 del libro, titulado «G estionar el lock-in», los autores analizan las diferentes estrategias de cálculo competitivo que, en el lábil límite fronterizo con la competencia desleal, sirven a las empresas que venden productos informacionalmente ricos en un entorno industrial caracterizado por los efectos externos de la conectividad reticular y la complementariedad software/hardware, para atrapar al mayor número de consumidores posible durante el mayor plazo de tiempo. D e entre los diversos modelos de estrategia empresarial tratados en la obra, uno de los más notables, en razón de su ambigüedad legal, es el sistema de tarifación conocido como «de precios de Ramsey», en el que la discriminación de precios es empleada por el monopolista exclusivamente como medio para recuperar los costes fijos de desarrollo 0 «costes de la primera copia». La lógica de los precios de Ramsey intersecta en muchos casos con otra lógica más perversa, reconocida en al gunos casos como abiertamente ilegal: la de los Ilamados «precios depredadores» (predatory prices), según la cual un monopolista vende de forma transitoria a precios por debajo de sus costes con el objetivo de eliminar a la competencia y cosechar más tarde rentas de monopolio (véase más abajo).

El capítulo 9, titulado «C omo librar una batalla de estándares», es particularmente interesante desde el punto de vista de la apertura moral del cálculo mercantil en el ambiente explícitamente probabilista de un mundo conéctico. Las empresas que compiten en los mercados de información pugnan entre sí para promover sus propias y especiales rutinas de producción y formatos de producto al estatus de «estándares industriales de facto». Para conseguir tal propósito, despliegan todo el arsenal conocido de estrategias y tácticas de actuación en un contexto de «batalla de estándares» (Besen y Farrell, 1994). D esde los preanuncios de lanzamiento hasta la concesión gratuita de licencias de uso, la firma de acuerdos de exclusividad con los principales proveedores, la integración vertical de esos mismos proveedores o la esponsorización de «tecnologías puente» para adaptar entre sí diferentes sistemas propietarios, el resultado a largo plazo de las estrategias competitivas puestas en marcha tanto por las cor- 
poraciones tradicionalmente dominantes como por los recién llegados al negocio para adaptarse a la combinación de estrechamiento y proliferación de nue vos mercados a la vez más segmentados y más interconectados, tiende preferentemente a la homogeneización más que a la diversidad.

\section{La ciencia social en una sociedad desencantada (II): el análisis de redes sociales}

También la academia sociológica se ha lanzado a enmendarles la plana a los modelos microeconómicos clásicos de mercados perfectamente competitivos con precios de equilibrio eficientes, identificando y cuantificando una serie de nuevas variables independientes de naturaleza estructural y cultural. Uno de los factores «socioeconómicos» más publicitados por estas investigaciones han sido los recursos de capital social poseídos por los competidores en un sistema de mercado. La sociología estructural de los mercados intenta de este modo hacer operativa, traduciéndola a conceptos analíticos bien delimitados, susceptibles de formalización matemática y medición estadística, la conocida concepción del «empotramiento social» (social embededdnes) de las actividades de producción e intercambio económico desarrollada por Karl Polanyi como parcela de su teoría general de los modos históricos de institucionalización social de actividad económica (Polanyi, 1976).

El trabajo pionero en este campo es un artículo ya clásico de $\mathrm{H}$ arrison C . White, profesor de la Universidad de Columbia, publicado en 1980 en el Ame rican Journal of Sociology, «W here D o M arkets C ome From?» [«iD e donde vie nen los mercados?»] (W hite, 1980). En este trabajo, W hite elabora un modelo de redes sociales para describir un fenómeno bien conocido por los gestores empresariales que tratan de hacer negocio en los mercados industriales: cuando la información material disponible sobre las expectativas de precios y calidades de los productos de la competencia es demasiado ambigua o incierta, la dinámica de mercado puede ser modelizada con bastante precisión en la forma de un proceso imitativo en el que cada uno actúa como ve actuar a los demás. M ás específicamente, los agentes en este contexto emplean su conocimiento «indígena» de la estructura social de la competencia mercantil a nivel local (alianzas formales, acuerdos informales, proyectos de colaboración o conexiones personal es entre miembros de distintos consejos de administración [interlocked directoriates] de las que se tiene conocimiento) como la mejor aproximación posible a la información económica relevante sobre «o que los demás tienen pensado hacer». (V éase Bourdieu, 2003: 256)

Sobre la base del trabajo de W hite, una serie de investigaciones posteriores han ofrecido explicaciones «racionales», en términos de «inteligencia social» de los actores económicos, de algunas de las más flagrantes anomalías competitivas observadas por la teoría económica en el comportamiento real de los mercados modernos. En uno de los ejemplos más logrados de este programa investigador, Joel Podolny, profesor de sociología de la Escuela de Administración de Empresas de la U niversidad de Stanford, ha descrito un mecanis- 
mo estructural capaz de dar cuenta del origen de la férrea estructura jerárquica del mercado de suscripciones bancarias de nuevas emisiones de títulos de la deuda pública estadounidense. Podolny (1993) emplea una variante del modelo del coste de señalización cualitativa en mercados con información imperfecta desarrollado por el economista M ichael Spence, profesor de la Universidad de $\mathrm{H}$ arvard y Premio $\mathrm{N}$ obel de Economía de 2001, en la que se incluye una medida indicativa del estatus social de una empresa como «proxy» de la variable «reputación empresarial». Regresionando diferentes indicadores de estatus, como las veces que un banco de inversiones aparece como «primer asegurador» en anuncios públicos de suscripción sindicada de valores durante los últimos diez años o el número de artículos que se le han dedicado en The Wall Street Journal, sobre diferentes índices de poder de mercado, como la capitalización bursátil, el volumen de facturación o los beneficios antes de impuestos durante el mismo período, el autor valida la hipótesis siguiente: la magnitud de recursos invertidos por los bancos en el cuidado de su reputación social explica la segmentación del mercado sobre la base de signos de distinción cualitativa con un al to coste de producción.

Un segundo tipo de aproximación socioestructural al comportamiento estratégico de las empresas en un entorno de competencia imperfecta, parte de la idea de que las redes sociales, además de como mecanismos de imitación que permiten reemplazar una información ausente o poco fiable, pueden funcionar también como mecanismos alternos de «clausura» (closure) e «intermediación» (brokerage) que actúan en origen sobre el propio flujo de información mercantil y en destino sobre los usos de esa información en los procesos de toma de decisiones. Este enfoque considera que, supuesto un determinado coste temporal de la comunicación del que deriva el carácter no simultáneo del acceso a la información por parte de diferentes individuos o empresas, los costes de búsqueda y los beneficios de anticipación que suelen obtener quienes disfrutan de un acceso temprano a la información pueden ser calculados a partir de un conocimiento de la morfología reticular de los grupos social es entre los cuales circula ésta. Este método de análisis es el más empleado por los trabajos de investigación que versan sobre el diseño de estrategias eficientes de gestión empresarial del capital social. Su variedad más conocida y de más impacto en la práctica de los administradores empresariales es la teoría de los «agujeros estructurales» (structural holes) desarrollada por Ronald S. Burt, profesor de estrategia empresarial en la Escuela de N egocios de la Universidad de C hicago.

D efinido en términos simples, un agujero estructural es aquella configuración reticular en la cual una persona está vinculada con otras dos personas, las cuales no están directamente conectadas entre sí $^{2}$ El potencial benéfico 0

2. « La participación en y el control de la difusión de información es el fenómeno subyacente al argumento de la acumulación de capital social bajo la forma de agujeros estructurales. Este argumento describe el capital social como una función de oportunidades de intermediación, y se apoya sobre conceptos de redes que surgieron en la literatura sociológica duran- 
«de negocio» que contiene esta situación puede ilustrarse con el siguiente ejemplo (adaptado de Baker, 2001: 11):

1. Al entrar en tu empresa actual estuviste destinado en el departamento de producción y conociste a Carlos, que todavía sigue trabajando allí.

2. Antes de entrar en esta empresa, estuviste una corta temporada de prácticas en una empresa de trabajo temporal donde conociste a Pili, que ahora casualmente trabaja en el departamento comercial de la misma empresa donde trabajáis tú y C arlos.

3. Carlos y Pili no se conocen entre sí, de hecho ni siquiera tienen constancia de que el otro existe.

4. En un momento dado te enteras de que Pili está buscando información sobre las necesidades de un nuevo cliente.

5. Tú sabes que, antes de entrar en la empresa, Carlos estuvo trabajando para el cliente con el que ahora está negociando Pili.

6. Si pones en contacto a Pili con C arlos para que éste le cuente lo que sabe de ese cliente, lo que estás haciendo es «tapar» un agujero estructural y, de hecho, proporcionar valor añadido al proceso productivo de tu empresa.

Un interesante caso de estudio sobre las tendencias actuales en el dominio de las prácticas de gestión empresarial inspiradas en la teoría reticular del capital social nos lo proporcionan los documentos de trabajo sobre estrategias de gestión del capital social accesibles a través de la página web de la empresa H umax Corporation, especializada en el diagnóstico y la estrategia de redes sociales (www.humaxnetworks.com). (Esta consultora de gestión empresarial fue fundada por el sociólogo Wayne Baker, uno de los investigadores más prestigiosos en el campo de las redes sociales (véase supra la n. 1.) En su calidad de consejero delegado de H umax Corp., Baker ha planteado una estrategia de negocio que, a grandes rasgos, consiste en desarrollar herramientas originales de gestión empresarial sobre la base del cuerpo de investigación básica acumulado a lo largo de los últimos veinte años en el campo de la teoría y la empiria del análisis de redes sociales. ${ }^{3}$

te la década de 1970; sobre todo en el modelo de G ranovetter (1973) de la fortaleza de los vínculos débiles, el modelo de centralidad intersticial (betweenness centrality) de Freeman (1977), el argumento de Cook y Emerson (1978) sobre los beneficios de poseer un patrón exclusivo de intercambios, y la proposición de Burt (1980) sobre la autonomía estructural creada por las redes complejas. D e manera más general, el argumento de los agujeros estructurales mezcla las ideas sociológicas elaboradas por Simmel (1955 [1922]) y M erton (1968 [1957]) sobre la autonomía generada mediante afiliaciones en conflicto, con las ideas económicas tradicionales sobre el poder monopolístico y oligopolístico para producir modelos reticulares de la ventaja competitiva» (Burt, 2000: 8-9).

3. Véase la siguiente formulación sintética del proyecto empresarial de H umax: «Un creciente cuerpo de investigación muestra que el éxito en los negocios depende de la competencia técnica y de la capacidad para construir redes de relaciones sólidas. No basta con sobresalir 
En un breve documento de trabajo, titulado «N etworking: Folklore Versus Fact» [Reticular: mitos y realidades], Baker ofrece una contraposición entre las concepciones económicas al uso sobre las fuentes del éxito empresarial (capacidades técnicas, formación) y un conjunto de «hechos probados» de la investigación sociológica sobre las virtudes del capital social como motor de la competitividad en los negocios (Baker, 2000a). A saber: los directivos que poseen redes sociales bien diversificadas ascienden más rápidamente y a edades más tempranas, las personas que buscan empleo a través de contactos personales encuentran trabajos más satisfactorios y mejor remunerados, y los líderes que han de competir en entornos novedosos se desenvuelven mejor cuando poseen una base sólida de relaciones sociales.

Los servicios de consultoría ofrecidos por $\mathrm{H}$ umax se concretan en un conjunto de instrumentos al gorítmicos para el diagnóstico de redes, que incluyen técnicas de mapas de visualización, análisis de perfil y fórmulas de estrategia orientadas a la maximización del capital social contenido en una red personal. El principio de análisis formal que subyace en el diseño de las herramientas de gestión del capital social comercializadas por H umax es la teoría de los agujeros estructural es desarrollada por Ronald Burt para operacionalizar la hipótesis del capital social y obtener medidas aproximadas del volumen de recursos explotables que se acumula en pequeñas provincias locales de una red social como consecuencia de una determinada configuración morfológica a escala global. Los conceptos formales básicos de la teoría de los agujeros estructurales son traducidos por H umax Corp. al lenguaje directamente pragmático y utilitarista de la gestión organizacional y la estrategia empresarial. En un texto titulado «C ómo hacer los amigos adecuados dentro de tu empresa», Baker (2000b) presenta un decálogo de las reglas de oro del «reticulador inteligente», entre las que se incluyen las siguientes máximas:

- H az una lista de las relaciones - fuertes y débiles - que posees actualmente dentro de tu empresa.

- Evalúa el grado de diversificación de tu red entre vínculos fuertes y débiles, y entre vínculos ocasionales y relaciones estables a largo plazo.

- Lo más importante es identificar las carencias de tu red: ¿cuáles son sus puntos de fisura críticos? ¿Q ué relaciones te haría falta tener para conseguir la información o los recursos que necesitas para llevar a cabo tu tarea?

en la parte técnica de nuestro trabajo. D ebemos además sobresalir en el arte y la ciencia de la reticulación [networking] [...] Las competencias necesarias para construirse una buena red de relaciones pueden ser aprendidas por cualquier persona. Lo que usted necesita es disponer de información novedosa sobre la importancia y el poder de las relaciones sociales y asesoramiento específico sobre cómo utilizar esta información en la práctica. A lo largo delos últimos quince años he trabajo en un área de investigación sociológica conocida como "análisis de redes". Junto con otros muchos investigadores, hemos llevado a cabo importantes hallazgos científicos sobre cómo construir redes de relaciones eficientes, pero hasta ahora este cuerpo de investigaciones había sido mantenido en secreto [sic] dentro del mundo de los negocios» (Baker, 2000a: 1, 3). 
- Una vez que conoces los huecos que existen en tu red, debes comenzar a rellenarlos: muéstrate auténtico y genuino en el establecimiento de tus nuevas relaciones, pues el mejor modo de comenzar una relación es dejar que la conexión ocurra de forma espontánea. ${ }^{4}$

- Conviértete en un «hombre puente» (bridger), la persona que constituye el vínculo crítico entre dos grupos.

Veamos a continuación el resumen de un caso de consultoría concreto extraído de Baker (2000c: 3-4): el análisis de la red personal de un ejecutivo empresarial (un director financiero de sociedades de capital riesgo, fundador de una empresa de servicios informáticos de tamaño medio con sede en la conurbación de Chicago).

El análisis de los datos que permiten configurar el perfil de capital social contenido en esta red personal, obtenidos mediante la autoadministración de un cuestionario (denominado H umax Assesment) vía Internet, se estructura en torno a tres dimensiones autónomas: el tamaño de la red, su composición y su enfoque. ${ }^{5}$ En cuanto al tamaño de la red, el análisis concluye, para este caso, que la red personal podría llegar a ser más amplia. Por el contrario, el diagnóstico de su estructura indica que posee un alto potencial endógeno para la acumulación de capital social. «En sus respuestas a las preguntas del cuestionario [el sujeto] nombró a diecisiete personas, un poco más de la mitad de treinta, que es el máximo posible permitido por el formato [de cuestionario] aplicado. Si una gran parte de esas diecisiete personas hubieran estado a su vez conectadas entre sí, su red hubiese adolecido de una carencia de agujeros estructurales, y su tamaño «efectivo» hubiera sido bastante peque ño. Pero el hecho de que la mayoría de los nombres no se hallaban vinculados entre sí, indica que existen muchos agujeros estructurales.» 0 tros indicadores estándares de capital social - como el bajo «índice de densidad» de

4. Véase SCH ELER (2002). Sobre «el arte de la conversación» y la «autenticidad» personal del reticulador social profesional. Para un tratamiento en extenso de los dilemas fundamentales que el paradigma ingenieril de las redes sociales plantea a las categorías culturales tradicionales de autenticidad y espontaneidad, cf. BOLTAN SKI y CHIAPELLO (1999: cap. 7).

5. «M is trabajos de consultoría para clientes de $\mathrm{H}$ umax me han permitido caracterizar un total de veintisiete tipos diferentes de perfiles de capital social. En un extremo estaría la red de tamaño pequeño, homogénea, y focalizada internamente. Para este perfil el capital social existe sobre todo en la forma de confianza y cooperación entre una red muy densa de personas muy similares entre sí. Este tipo de red es bueno para construir lealtades grupales, identidad, y un sentido de propósito colectivo. Sin embargo es poco adecuada para obtener información u otros recursos, o para influenciar a personas de fuera de la red... En el otro extremo tenemos el tipo de red amplia, diversa y focalizada hacia el exterior. Este perfil representa la existencia del capital social en la forma de oportunidades empresariales. Existen abundantes «agujeros» entre las personas, de modo que puede crearse valor mediante la introducción de gente desconectada. Este perfil es bueno para obtener información nueva, para enterarse de nuevas oportunidades y para hallar recursos. Pero se adapta mal a la construcción del consenso... y suele ser fuente de conflictos y tensiones» (Baker, 2000c: 4). 
la red: 6 el número de vínculos reales sólo al canza el 16\% del número máximo de vínculos teóricamente posibles- señalan en la misma dirección.

Con respecto a la composición de la red, el eslogan de H umax Corp. sostiene que «la similaridad es el enemigo de la buena reticularidad», siendo, en cambio, la «diversidad» el síntoma mayor de una red de amplio alcance. En este caso, la red personal bajo estudio estaba compuesta de elementos muy diversos, tanto desde el punto de vista de la edad como del nivel educativo 0 del género; si bien desde el punto de vista racial imperaba la más completa homogeneidad. Tras el repaso de este segundo eje del examen de la red personal, el consultor apunta que «la composición global de la red [de nuestro sujeto] cae dentro de la parte media de nuestra escal a de diversidad. N uestro diagnóstico es que la estrategia de creación de val or en este caso debe basarse en la diversificación de los contactos».

En tercer lugar, el análisis del enfoque de la red traza una mezcla de actividades diversas y de «puentes» que permiten cruzar la divisoria entre vida laboral y vida familiar - el $24 \%$ de las personas de la red personal son miembros de la propia familia y sólo el 18\% son gente relacionada con el trabajo (un nivel por debajo de la media de su clase, según la escala estándar empleado por H umax). Por último, casi un $40 \%$ de los nombres de la red han vivido y trabajado en el extranjero, lo cual, interpreta el consultor, es un indicador fiable de que la red considerada posee un nivel de «enfoque global» superior a la media.

\section{3. ¿H acia un derecho civil de las redes sociales? El ejemplo de la jurisprudencia estadounidense sobre competencia ilícita en los mercados de red}

Los modelos de topología estadística y los más avanzados modelos de procesos dinámicos y flujos estocásticos en espacios reticulares, han carecido hasta hace muy poco de un lenguaje interpretativo propio que hiciera justicia a su enfoque estrictamente relacional y contingente 0 «desustancializado» del sujeto y la estructura social. Las más de las veces, el sentido práctico del trabajo de cálculo estadístico en un espacio de redes ha sido pervertido por la importación de vocabularios teóricos de ascendente estructuralista que, si bien permitían dar cuenta analítica de la estabilidad de ciertos patrones de orden calculable - así, los índices de centralidad reticular (Freeman, 2000) - específicos de un universo de relaciones entre relaciones, no hacían tanta justicia a la parte estrictamente indeterminista de los modelos más avanzados - que incluyen índices de dispersión poblacional (Granovetter, 1990) y complejidad jerárquica (Padgett, 1981) - , es decir, a aquello que de inherentemente aleatorio tiene la definición de los agregados sociales cuando su origen puede retrotraerse a las microdesviaciones incesantes que sufre una cadena de mensajes en su tortuo-

6. Cf. PIZARRo (1990: 63-68) y M o LINA (2001: 80-82) para diferentes formas de cal cular este índice. 
sa circulación a través de espacios multidimensionales de posiciones solapados entre sí.

A continuación trataré de mostrar, con un pequeño estudio de caso sobre la controversia jurídica en torno a la definición de las prácticas de competencia ilícita en los mercados de red, que, en realidad, el vocabulario categorial que mejor sirve a la comprensión de sentido común de estas nuevas formas de cálculo estratégico está siendo elaborado por los tribunales de justicia y las agencias de regulación pública, lugares sociales «híbridos» donde las prácticas especializadas de la investigación científica se mezclan de forma inextricable con las rutinas del trabajo administrativo bajo el signo distintivo de las urgencias temporales y las contingencias banales de la vida ordinaria (Lynch y Jasanoff, 1998).

\subsection{Identidad del producto y frontera entre mercados}

La pertenencia simultánea de la información factual a dos regímenes contrapuestos de regulación de la actividad económica, la esfera del intercambio privado donde se impone una estructura de incentivos económicos (como la definición de derechos de propiedad excluyentes) con el propósito de aumentar los niveles de producción, y la esfera de la redistribución pública, donde se imponen incentivos de signo contrario (restricciones y excepciones a la apropiación exclusiva) para tratar de aumentar los niveles de difusión, es el rasgo característico de todos los mercados donde se comercian bienes o servicios ricos en información. D esdee mercado editorial hasta el mercado farmacéutico, pasando por el mercado informático, el cinematográfico, el crediticio, el asegurador y, en general, todos los mercados de servicios de asesoría técnica y consultoría experta (fiscal, legal, psicológica, educativa, empresarial, etc.).

Una de las soluciones institucionales más ampliamente adoptadas a lo largo de la historia y a través de las culturas para contener, siquiera transitoriamente, el tipo característico de desorden entrópico - que los economistas neoclásicos Ilaman «imperfecciones» y los sociólogos de la N ueva Economía denominan «desplazamientos» (Boltanski y Chiapello, 1999: 402-412)continuamente generado por el arbitraje informacional de oportunidades de negocio en el enrejado infinitamente extenso y anidado de los mercados industriales contemporáneos, ha consistido en garantizar estatalmente a los agentes privados un conjunto especial de derechos de propiedad y control monopolístico con fecha de caducidad. La violación de estos derechos especiales de propiedad (como las patentes y los derechos de autor) constituye uno de los capítulos central es de una variedad mayor de faltas y delitos tecnoeconómicos: las infracciones cometidas contra las leyes de defensa de la competencia o leyes antimonopolio (anti-trust laws), como se conocen en los Estados U nidos. ${ }^{7}$ Además de las violaciones de patentes y derechos de autor y la piratería

7. Para consideraciones teóricas de fondo sobre la naturaleza económica, jurídica y política de la competencia mercantil, véase D EM SETz (1986). Para una introducción al desarrollo 
de marcas protegidas, la figura legal del «ilícito concurrencial» 0 «competencia ilícita» (que abarca un amplio espectro de responsabilidades civiles y penales) subsume prácticas tan diversas como los fraudes en la calidad, la connivencia empresarial para la fijación de precios abusivos y la restricción de la producción o el llamado «abuso de posición dominante», cometido por una empresa líder que «apalanca» su poder de mercado para restringir aun más el margen de maniobra de sus competidores o bien para conquistar mercados de productos independientes. ${ }^{8}$

La famosa cruzada legal emprendida desde principios de la década de 1990 por el Departamento de Justicia de los Estados Unidos. contra el emporio empresarial del gigante informático M icrosoft, acusado de emplear prácticas ilegales de abuso de poder de monopolio y acaparamiento de mercado, condensa de forma inmejorable varios de los más importantes dilemas que han de enfrentar las doctrinas clásicas del derecho civil y mercantil para dar respuesta a este nuevo paradigma de análisis positivo del orden social que representan el análisis económico de la información y el análisis sociológico de las redes. ${ }^{9}$

conjunto de las leyes antitrust en los Estados U nidos y de la moderna teoría de la organización industrial, véase Kovacic y SH APIRO (2000), y FERN ÁN DEZ O RD Ó ÑEZ (2000: cap. 9) sobre la experiencia española más reciente en materia de política de defensa de la competencia.

8. En estos y otros casos de conducta anticompetitiva, la infracción del procedimiento legalmente establecido exige que los delincuentes - ipero también los policías!- posean un conocimiento experto de al to nivel de las sutilidades de los modelos microeconómicos de la teoría de los juegos y del diseño de mecanismos para incentivar el revelamiento de información en contexto estratégico - justamente el penúltimo de los campos de investigación bendecidos por la mano sagrada del Comité N obel de la Academia Sueca. (Los trabajos seminales publicados por el economista británico James M irrlees sobre la aplicación de modelos de incentivos económicos eficientes para la extracción de información privada a la resolución del problema del nivel óptimo de presión fiscal en entornos donde los contribuyentes pueden ocultar al fisco información sobre sus ingresos, y por el matemático canadiense W illiam Vicrey, que diseñó diversos mecanismos de subasta para la asignación óptima de recursos en condiciones de información asimétrica, les valieron la concesión del Premio $\mathrm{N}$ obel de Economía de 1996.) En este sentido, por ejemplo, el economista Paul K lemperer, profesor de la U niversidad de 0 xford y experto de reconocido prestigio en el diseño de mecanismos económicos de incentivos a la revelación de información, salió a la arena pública en el otoño de 2000 para ofrecer «argumentos científicos sólidos» para exigir en los tribunales responsabilidades legales al gobierno italiano por la negligencia profesional de los expertos que diseñaron un procedimiento de subasta ascendente y lo emplearon para resolver un concurso de concesión de licencias de telefonía móviles con tecnología U M TS, a sabiendas de que el pequeño número de licitadores presentados a concurso hacía el procedimiento extremadamente vulnerable a estrategias de manipulación (Klemperer, 2000).

9. Para una divertida introducción al análisis jurídico-económico del caso M icrosoft, véase SaLa I M ARTín (2002). Para una presentación más técnica de la literatura económica especializada sobre el análisis antitrust de las industrias de red, cf. SH APIRO (1996 y 2000), y KaTz y SH APIRO (1998) para el caso particular de los mercados de programas informáticos. Una visión panorámica de las innovaciones introducidas por el D epartamento de Justicia de los Estados U nidos en materia de regulaciones antitrust específicamente adecuadas a la estructura competitiva de las nuevas industrias de la información digital puede encontrarse en KLEIN (1998). 
En 1994 el D epartamento de Justicia de los Estados Unidos había abierto diligencias contra M icrosoft por la estructura de precios empleada para licenciar su sistema operativo M S-D OS a los fabricantes de ordenadores. En este sistema, conocido popularmente como de «icencias por procesador», los comerciales de M icrosoft calculaban la tarifa que había de pagar el fabricante de hardware por cada copia de software instalada sobre el número total de orde nadores producidos y no sobre el número de aquéllos en los que efectivamente había decidido instalar M S-D O S, con lo cual casi se obligaba al fabricante a acabar instalando el programa de $\mathrm{M}$ icrosoft en todos sus ordenadores, ya que de hecho estaba pagando como si así fuese. Como consecuencia de estas primeras diligencias, M icrosoft abandonó en buena parte los acuerdos de exclusiva que bloqueaban a sus competidores. La segunda demanda presentada por el D epartamento de Justicia en mayo de 1998 hacía referencia a un tipo muy distinto de práctica comercial conocido como «amarramiento» (tying), mediante la cual M icrosoft distribuía un paquete de software propietario para hojear las páginas web colgadas en Internet (el browser «navegador» bautizado como «Explorer») incluido como parte del escritorio de su sistema operativo M S-W indows, aprovechando aś la amplia base instalada del mismo.

El enjuiciamiento de la legalidad de la estrategia de amarramiento del nave gador Internet Explorer al sistema operativo W indows no es en absoluto sencillo. D e hecho, ha obligado a los altos tribunales americanos a plantearse preguntas de corte filosófico tales como qué es realmente un «sistema operativo», qué es realmente un «navegador de Internet» 0 dónde está el límite del producto «sistema operativo» y cuál es la frontera que separa su mercado específico del mercado de «navegadores de Internet».

Al margen de las tópicas declaraciones programáticas sobre la defensa de la innovación y la defensa de los consumidores (tanto M icrosoft como sus demandantes se presentaron ante el juez como empresas innovadoras que buscan proporcionar cada vez mayor valor añadido a sus usuarios), fue la controversia más concreta sobre la identidad técnica de los productos, y su correlato lógico, el problema del trazado de fronteras estables entre mercados diferenciados, la cuestión sobre la que giró la parte más importante del debate público sobre el carácter legal o ilegal, justo o injusto, de los métodos de gestión estratégica de su red de productos instalados empleadas por M icrosoft para conservar y ampliar su poder de monopolio en el mercado de los programas de ordenador.

Uno de los eslabones clave de la disputa sobre la identidad separada del sistema operativo y el navegador de $M$ icrosoft y de sus respectivos mercados, son los llamados W indows API (por Application Program Interfaceso interfaces de aplicación), la parte del código del programa W indows que permite que los programas producidos por otras empresas de software, como el procesador de textos W ordPerfect o la hoja de cálculo Lotus 1-2-3, puedan funcionar en su entorno. Aun cuando en numerosas declaraciones públicas, el presidente de M icrosoft, W illiam (Bill) Gates ha sostenido que su sistema operativo posee un carácter «abierto», en el sentido de que los proveedores de software indepen- 
dientes tienen libre acceso al código de las API deW indows, cuyo conocimiento les resulta imprescindible para hacer plenamente compatibles sus productos con el sistema operativo estándar de M icrosoft, los programadores de aplicaciones en plantilla de M icrosoft - y muy especialmente los desarrolladores del paquete ofimático O ffice- siguen siendo los primeros en enterarse de los cambios que se prevee introducir en el código de las API, con lo que siguen disfrutando de una ventaja comparativa para adaptar sus aplicaciones a la plataforma W indows. La defensa adoptada por los analistas económicos y los asesores legales de M icrosoft contra esta línea de ataque ha consistido en argumentar que la conservación de un cierto poder de timing, o control sobre el ritmo temporal de difusión de las modificaciones en los API es fundamental para la buena marcha del proceso innovador encargado del mejoramiento de su sistema operativo. A lo cual los desarrolladores de software independientes respondieron denunciando a su vez que al gunas de las funciones más «sabrosas» de sus programas sufren continuamente la amenaza de ser integradas por M icrosoft como parte de su sistema operativo (Varian y Shapiro, 2000: 191). ${ }^{10}$

En el primer gran asal to para decidir esta disputa (que resultó fallido) ${ }^{11} \mathrm{el}$ juez federal Thomas Jackson sentenció, a principios de noviembre de 1999, que la práctica del tying habría permitido efectivamente a M icrosoft apalancar de forma ilícita un enorme poder de mercado lícitamente obtenido en el segmento de los sistemas operativos (con un $95 \%$ de la base total instalada a mediados de la década de 1990) para barrer definitivamente del mapa a su principal competidor (el browser N avigator propiedad de la empresa N etscape) en la nueva área de negocio de los programas de navegación en Internet.

\subsection{La manipulación estratégica de los mercados en red}

Como han apuntado Kovacic y Shapiro (2000: 58), el esqueleto analítico de una buena parte de los argumentos legales esgrimidos por el Gobierno estadounidense como parte acusadora en la segunda demanda contra M icrosoft

10. Una solución alternativa a la controversia judicial sobre la naturaleza abierta o propietaria del código Windows API ha sido propuesta por la comunidad de programadores informáticos que trabajan en el entorno del sistema operativo GN U/Linux, que se distribuye bajo una licencia especial denominada GPL (G eneral Public License), que obliga a distribuir el código fuente de cual esquiera programas cuyo desarrollo incluya una parte del código GNU I Linux protegido mediante la licencia G PL. Los programadores del entorno Linux han puesto en marcha recientemente un ambicioso proyecto denominado WINE, cuyo objetivo es «clonar» - v. g. replicar, empleando técnicas de ingeniería inversa, un código propietario protegido por las leyes de patentes o bien, como parece ser aquí el caso, escondido bajo el frágil manto legal de los secretos industriales- el código W indows API para hacer todas las aplicaciones escritas para W indows plenamente compatibles con el sistema operativo «ibre» GN U/Linux (Wayner, 2001: 423-24).

11. En junio de 2001, la revisión de la sentencia por la Corte Federal de Apelaciones estadounidense, aun cuando ratificó las partes de la sentencia que hacían alusión al uso ilegal del poder de monopolio por parte de M icrosoft, decidió devolver de nuevo el caso al juez Jackson para un nuevo examen (cf. la información del diario El País de los días 29 y 30 de junio de 2001). 
contiene una amplia dosis de conceptos y al goritmos de equilibrio estratégico ela borados por la segunda (juegos con información imperfecta) y la tercera (juegos dinámicos evolutivos) generaciones de investigaciones sobre la aplicación de la teoría matemática de los juegos al estudio de la competencia económica.

A mediados de la década de 1980, los últimos avances técnicos de la teoría de los juegos de competencia económica habían empezado ya a emplearse para fundamentar de forma objetiva algunos viejos argumentos legales en materia de defensa de la competencia. Uno de los precedentes más señalados en el establecimiento de esta tendencia fue la sentencia de la Corte Suprema de los Estados U nidos en el caso de Eastman Kodak C 0. vs. Image Technical Services (1992). Kodak fue acusada de monopolizar el mercado de los servicios posventa de sus propias fotocopiadoras. Inicialmente, un tribunal de primera instancia decidió en favor del demandado arguyendo que, puesto que su cuota de mercado en las ventas de nuevas fotocopiadoras sólo llegaba al $20 \%$, era irracional para la propia empresa imponer restricciones en los servicios posventa a los propietarios de su base instalada. En su revisión de la sentencia, la Corte Federal de Apelaciones resolvería posteriormente devolver el caso al tribunal de instancia para un nuevo examen. En la última ronda de apelaciones, la Corte Suprema revirtió la decisión condenando a Kodak. Los fundamentos jurídicos de la sentencia de la Corte Suprema integraban en su núcleo central una teoría extendida del poder de mercado apoyada sobre las nociones económicas no clásicas de «información asimétrica» y «atrapamiento de fase» (phaselock-in). En concreto, la sentencia hacía uso de un abstruso tecnicismo microeconómico, el concepto de «oportunismo de base instalada», para demostrar que ciertas estrategias aparentemente irracionales en el mercado de servicios complementarios de asistencia técnica pueden ser consideradas perfectamente racionales cuando se las evalúa desde el punto de vista de una estrategia más general orientada a hacerse con una masa crítica de clientes cautivos que permita barrer el mercado en un futuro próximo. ${ }^{12}$

Pero no ha sido hasta bien avanzada la década de 1990 cuando la doctrina antitrust de los tribunales estadounidenses ha sufrido la irrupción en masa de los nuevos model os económicos de información imperfecta y estrategias evolutivamente estables (Foster y Young, 1990). Esta penúltima ofensiva con-

12. Una pregunta pertinente que cabría formular contra este argumento sería la siguiente: icómo de próximo ha de ser ese futuro para que la estrategia de manipulación del mercado pueda calificarse como racional y no como descabellada? En el caso M atsushita Electric Industrial Co. v. Zenith Radio Corp (1986), la Corte Suprema estadounidense. argumentó que un monopol ista que emplee precios depredadores tiene pocas probabilidades de sostener su monopolio en el futuro durante el tiempo necesario para recuperar las pérdidas incurridas al vender en el presente por debajo de sus costos. En términos analíticos, en efecto, la estimación precisa de los parámetros cronológicos que deslindan el corto del medio y el largo plazo en el análisis del sostenimiento de una determinada fórmula de fijación de precios es un elemento de prueba crucial, aunque en modo alguno definitivo, para establecer la factibilidad del ilícito en este tipo de estrategias empresariales orientadas a influir sobre las expectativas de los consumidores. 
ceptual del análisis económico sobre la hermenéutica legal ha permitido dar carta de natural eza a las controvertidas realidades empíricas que se esconden tras al gunos calificativos, como «disuasorio» (así «barreras de entrada disuasorias»), «creíble» («amenazas creíbles» 0 «compromisos creíbles») 0 «depredador»- cuando se habla de «precios depredadores»- que nunca habían gozado de excesivo predicamento entre los juristas.

La ponderación legal de tecnicismos adjetivos de tan amplio espectro empírico ha estado de este modo sujeta en los últimos años a los vaivenes académicos (bastante caprichosos en sí mismos) ${ }^{13}$ de la corriente central de la teoría microeconómica de la organización industrial. M ientras que, a lo largo de la década de 1980 y hasta la primera mitad de la de 1990, conceptos teóricos como el de "costos variables medios», acuñado por los autores de la N ueva Escuela de Chicago en los años sesenta, permitieron justificar decisiones judiciales que negaban la factibilidad de los precios depredadores, considerándolos una estrategia irracional o directamente suicida desde el punto de vista de un monopolista (Posner, 1998: 290-294), con el ascenso académico del enfoque de teoría de juegos como núcleo duro de la teoría de la competencia imperfecta, las autoridades anti-trust de los Estadus U nidos volvieron a declarar admisible el argumento de que la estrategia (ilegal) de precios depredadores tiene sentido en un contexto de información imperfecta y costes de señalización cualitativa. ${ }^{14}$

\section{Azar, persona y responsabilidad: un triángulo complicado}

$M$ ientras que los economistas matemáticos no han solido mostrar grandes vel eidades epistemológicas en la justificación de sus programas de investigación, no puede decirse lo mismo de los sociólogos matemáticos. Valiéndose de un argumento de corte historicista, los fundadores del programa de investigaciones sobre análisis matemático de redes sociales a principios de la década de 1970, lo definieron como el tipo de análisis científico que mejor se adecuaba al estudio de sociedades abiertas donde la más pura casualidad es uno de los componentes constitutivos de la estructura social. «D isponemos de una lista creciente de pruebas empíricas relativas a los efectos y a la frecuencia de los "accidentes" o de los "azares" en el funcionamiento efectivo de las sociedades", escribían H arrison W hite, Scott Boorman y Ronald Breiger en uno de los artí-

13. Véase M IROWSKI (2002) para una prolija reconstrucción histórica de las trayectorias tecnológicas heterogéneas que han confluido en la conformación del análisis económico contemporáneo como ejemplo modélico de «ciencia cibórica».

14. Si bien con importantes matices técnicos - no exentos de ambigüedad en cuanto a su contrastación empírica (cf. supra la n. 12) - como el requisito, subrayado por KovACIC y SH APIRO (2000: 55), de que los potenciales nuevos entrantes en el mercado «deben poseer una memoria suficientemente larga» (mi énfasis) de la historia de «amenazas creíbles» (en forma de ventas por debajo de sus costes de producción) hechas en el pasado por el monopolista para desanimar los intentos de entrada de nuevos competidores. 
culos más influyentes de este programa (W hite, Boorman y B reiger, 1976: 733). Con el tiempo, la justificación historicista fue dejando paso a otra de tipo naturalista o «antropológico» que esgrimía la eficacia del análisis de redes sociales como la forma más natural de estudiar los mecanismos conexionistas y los procesos de «emergencia estructural » que están en el origen de agregados sociales característicos de muy diferentes épocas y culturas. ${ }^{15}$

A principios de la década de 1990 , el profesor $\mathrm{H}$ arrison C. White, pione ro y uno de los máximos exponentes del análisis de redes sociales, publicó una extraña (por altamente idiosincrásica) y en su momento no muy influyente obra titulada I dentity and Control. A Structural Theory of Social Action [Identidad y control. Una teoría estructural de la acción social] (W hite, 1992). En esta obra, W hite se imponía la hercúlea tarea de codificar un sistema de categorías conceptuales y fenomenológicas fundamentales para conferir sentido epistemológico al sofisticado aparataje metodológico y tecnológico con el que han hecho fortuna los investigadores de las redes sociales. «L os dos conceptos básicos de esta teoría son la identidad y el control, la primera se desencadena en forma de proyectos para conseguir el segundo por la acción de contingencias que conectan el mundo físico con el mundo social» (W hite, 1992: 16). El sistema teórico de W hite se ofrece, al mismo tiempo, como una herramienta particularmente adecuada para la descripción formal de buena parte de las novedades organizacionales más importantes producidas por la fábrica social contemporánea, tal como han sido identificadas por otras variedades de sociología empírica (e. g. nuevos patrones de organización productiva y administrativa empresarial, nuevos modelos de acción colectiva o de intervención estatal, nuevas formas de construcción de identidades personales y de formación de vínculos fiduciarios). Resumo brevemente a continuación los principios teóricos básicos sobre los que se erige este modelo eminentemente pos-estructuralista del orden social.

En el nivel estático, el análisis de las estructuras que propone W hite apuesta decididamente por la utilidad metodológica del principio minimalista de la invarianza de escala o simetría fractal, traducida aquí como 'autosimilaridad' entre diferentes niveles de agregación estadística de un mismo fenómeno sociológico. ${ }^{16}$ En segundo lugar, el estudio dinámico de los procesos sociales se cen-

15. BoLTAN SKI y C HIAPELLo (1999: 229-230) han señalado que, mientras que el enfoque historicista puede ser criticado por su carácter ad hoc, por tanto, por una falta de consistencia antropológica u ontológica, el problema epistemológico que plantea el enfoque naturalista es el de la inconsistencia entre las categorías del analista y las categorías de los actores.

16. El propio Benoît $M$ andelbrot, el polimatemático que convirtió un conjunto de divertimentos y curiosidades matemáticas en el sistema de la «geometría fractal», ha reconocido su deuda con un comentario que le fue formulado por W hite, en el contexto de un seminario sobre econometría impartido por el primero en la Universidad de $\mathrm{H}$ arvard en el curso 196263 , sobre la necesidad de enfatizar la diferencia entre el principio fractal de las simetrías de escala, de origen específicamente científico-social, y los criterios de invarianza al uso en aquel momento en el campo de la física, como el principio de relatividad ( $M$ andelbrot, 1997: 103). 
tra en el grado de flexibilidad que permite a la acción social intencional el juego de las categorías embedding-decoupling ('incorporación-desincorporación') (W hite, 1992: 17). El análisis categorial de la dinámica de las redes sociales gira de este modo en torno a la potencia creadora del concepto central de contingencia. La contingencia, esto es, la casualidad considerada como realidad objetiva e ineludible, es la pieza que articula la estática comparada de las autosemejanzas escalares de la organización social con la dinámica de «proyectos de control» sucesivamente emprendidos por las «identidades» para descubrirse, estabilizarse y extenderse.

La de contingencia es, pues, en este sistema teórico, una categoría ontológica: el puente de unión entre el espacio-tiempo del entorno biofísico y el espaciotiempo autónomo del mundo de la organización social. «Los proyectos de control son respuestas que dan las identidades a las contingencias estocásticas interminables a las que deben añadirse otros proyectos de control.» (Ídem: 9). El mundo del trabajo, que ofrece la principal superficie de contacto entre la frontera del mundo social y la del mundo físico, es considerado por W hite la principal fuente de aporte de contingencias o indeterminaciones biofísicas, necesarias para el detonamiento del cambio social (ídem, 111).

Esta teoría estocástica del control y la identidad social alcanza una de sus cotas más radicalmente posmodernas en el trabajo de desconstrucción fenomenológica que lleva a cabo de forma específica sobre el concepto humanista clásico de persona. Tradicionalmente considerada como «precondición límite» para la construcción teórica de las ciencias sociales, la persona ha pasado en manos de los teóricos de las redes sociales a convertirse en un mero concepto teórico-social de rango medio. En efecto, a diferencia de la gran mayoría de los model os explicativos disponibles actual mente en el campo de la investigación social, donde la existencia de la persona en tanto que «átomo incuestionado» se da por supuesta, W hite no concibe una ciencia social plenamente desarrollada que no asimile la persona como «una encarnación particular de una clase de actores socialmente construidos, y no la única clase» (ídem 196). Y desde este punto de vista, su existencia real es algo que «debe ser explicado». Pero para una teoría del orden social que se define a la vez como relacional y estocástica, la explicación de todo hecho social implica «que deben ser especificados aquellos contextos de análisis en los que [tal hecho] puede, podrá o no podrá ser introducido» (W hite, 1992: 196). El nuevo concepto de persona al que apunta este método de razonamiento es consistente con un modelo de ciencias sociales y cognitivas que acepta todas las implicaciones del catecismo antisubstancialista e indeterminista de la matemática, la física y la biología de finales del siglo xx: ${ }^{17}$ « as personas al canzan el ser y vienen a formarse como sola pamientos entre identidades adscritas a diferentes poblaciones de redes.» (ídem)

17. Para un desarrollo en profundidad del modelo «conexionista» de «yo fragmentado» 0 «persona sin yo» que se ha convertido en el paradigma dominante de las ciencias cognitivas durante la última década, cf. Varela, Thompson y Rosch (1992: parte tercera). 
Crecientemente confrontadas con discursos públicos que tratan sobre las identidades personales como subproductos que surgen de los deefases entre los proyectos de control di sciplinario y las contingencias contextuales «que recorren las historias de las redes y sus ecologías poblacionales» (ídem 197), las categorías estatutarias heredadas del derecho civil y penal clásico ( «responsable directo», «responsable subsidiario», «autor material», «coautor», etc.) basadas en una concepción substancialista de la «persona jurídica», han dejado de ofrecer apoyos estables para la formación del juicio concreto, convirtiéndose ellas mismas en convenciones problemáticas, susceptibles de ser relativizadas como «entidades emergentes, contingentes y contextualizadas». 0 bjeto, por tanto, al igual que los entes empíricos sobre los que juzgan, de disputa continua sobre los límites de sus «dimensiones propias».

Bien es verdad que tampoco la técnica matemática - ya sea en sus modalidades algorítmicas clásicas o en aquéllas de carácter complejo y no lineal- se ha mostrado capaz de resolver las aporías legales y morales que plantea la doble naturaleza, mercantil y política, de la información en tanto que hecho social material (Boyle, 1996: 29). Y ello porque el análisis formal de la naturaleza de la información en tanto que mercancía comerciable incurre de forma inevitable en las conocidas paradojas epistémicas con las que habían topado anteriormente tanto el análisis lógico-matemático, que intentaba penetrar en el contenido informacional de «secretos perfectamente guardados» (C aballero, 1996: 14-22), como el análisisfísico-ingenieril, que intentaba precisar el contenido energético de la información perfectamente comunicable (Landauer, 1991). La microeconomía moderna no ha hecho sino traducir parcialmente estos resultados límite, casi metafísicos, de la exploración científica básica sobre Ios límites fundamentales del mundo formalmente inteligible, al lenguaje específico de la competencia mercantil imperfecta en presencia de asimetrías de información (Akerlof, 1987): el licitador sólo puede conocer el valor económico real de la mercancía por la que puja una vez que tiene acceso a ella, pero si el vendedor le da acceso gratuito a ella para fines de inspección perderá de golpe todo el valor económico que le había asignado inicialmente a su mercancía (D avid y D asgupta, 1994: 496).

D e suerte que, enfrentados a la extraña fenomenología aleatoria de proyectos de acción flexibles, gestionados casi en tiempo real, orientados al futuro aunque con horizontes temporales de muy corto plazo, y coordinados de manera distribuida a través de redes locales radical mente opacas a los análisis de tipo global, las construcciones morales clásicas del derecho penal y civil, donde las categorías matrices de «causalidad», «imputabilidad» y «responsabilidad» se apoyan cada vez más frágilmente en pruebas de fiabilidad estadística, ${ }^{18}$

18. En otro trabajo (I zQUIERD o, 2002b) he intentado verificar esta hipótesis para un caso de estudio particular: el del «auge y caída» de un sofisticado tipo de estrategias de negociación bursátil (las denominadas «operaciones de valor relativo») que alcanzaron gran éxito en la industria global de derivados financieros a mediados de la década de 1990. 
afrontan en el momento actual serios problema de legitimidad. En este sentido, cabe reseñar final mente la propuesta de los sociólogos franceses Luc Boltanski y Eve Chiapello, quienes, en una importante obra titulada EI nuevo espíritu del capitali ismo (vid. Izquierdo, 2002c para un estudio crítico de la misma), han sostenido que el distintivo estilo posgarantista que transpiran una serie de líneas recientes de innovación procedimental en diferentes ámbitos de regulación legal ${ }^{19}$ podría ser la punta de lanza del proceso de conformación futura de un nuevo régimen de «justicia conexionista» que trataría de «conferir a las redes un estatuto legal con el fin de limitar las posibilidades de oportunismo y de explotación conexionista que se observan actualmente, pero sin volver a imponer formas jurídicas rígidas que las harían inoperantes porque no captarían la especificidad de las nuevas configuraciones» (Boltanski y Chiapello, 1999: 474-75).

\section{Conclusión}

En el capítulo introductorio de un manual práctico sobre estrategias corporativas y personales de gestión de los recursos de capital embebidos en las redes de interacción social, nuestro viejo amigo el sociólogo Wayne Baker exhibe el ya vasto corpus acumulado de investigaciones académicas del análisis de redes sociales como una especie de certificado de "garantía de validez científica» para los servicios de consultoría estrategia que comercializa su empresa, H umax Corp.:

Los principios sobre el poder del capital social que estoy a punto de compartir con usted se basan en evidencia dura - hallazgos científicos de estudios cuantitativos llevados a cabo por científicos sociales profesionales (sociólogos, psicólogos, economistas y politólogos). El concepto de capital social posee una larga historia intelectual, y por tanto esta obra práctica reposa sobre bases académicas sólidas. A lo largo de los últimos veinte años, los científicos sociales han documentado ampliamente los beneficios del capital social, tanto para los individuos como para las organizaciones. (Baker, 2001: 10)

19. Entre la causuística de proyectos independientes de «legislación sobre las redes» que mencionan los autores, se incluyen desarrollos tan heterogéneos como la institucionalización del «principio de precaución» en la legislación sanitaria y medioambiental, la formalización de un nuevo «derecho de actividad» en el ámbito laboral, o la propuesta de la llamada «tasa Tobin», un impuesto sobre las operaciones en los mercados internacionales de divisas, si bien en este último caso, el de los mercados financieros internacionales, la evolución general de las formas de regulación pública de la actividad privada discurre en realidad por otros derroteros muy distintos. Como he tratado de mostrar en otro sitio (Izquierdo, 2002a) el régimen internacional de prevención de riesgos de origen tecnológico en la industria global de servicios avanzados de intermediación financiera se decantó, a mediados de la década de 1990, por un modelo de autorregulación mercantil basado en la hipótesis de que la libre competencia entre sistemas propietarios de gestión de riesgos constituye la forma de precaución global más eficiente en un contexto de incertidumbre científica sobre efectos irreversibles a largo plazo. 
I maginadas como soluciones científicas provisionales a uno de los enigmas más ancestrales del orden social (el de cómo es posible coordinar de un modo descentralizado la acción autónoma de una masa crítica de individuos), las aplicaciones ingenieriles de «al gunos de los resultados cuantitativos más robustos» establecidos por el programa de investigaciones académicas sobre la topología y la mecánica estadística de las redes sociales, se han convertido en una fuente inagotable de nuevos problemas de orden público: desde nuevas formas «materiales» de manipulación de relaciones personales, hasta una tendencia generalizada a la intensificación tecnológica de las prácticas de competencia desleal y la «flex-plotación», o hasta un creciente uso estratégico de derechos fundamentales antagónicos como los de privacidad y libertad de información (Lessig, 2001).

C onsidero que este hecho es, en cualquier caso, plenamente consistente con la vieja conjetura de $\mathrm{H}$ arrison W hite (1973: 48), según la cual «os sistemas sociales [complejos] se muestran sorprendentemente inmunes a la manipulación deliberada, ya se base ésta en los descubrimientos del analista social o en los que llevan a cabo las personas en su vida cotidiana».

\section{Bibliografía}

AKERLOF, G. (1987). «El mercado de "limones": incertidumbre en la calidad y el mecanismo de mercado» [1970], Cuadernos económicos de ICE, 36, 37-48.

BAKER, W. E. (1984). «T he Social Structure of a N ational Securities M arket», Ame rican Journal of Sociology, 89, 775-811.

- (1990). «M arket N etworks and Corporate Behavior», American Journal of Sociology, 96, 589-625.

- (2000a). N etworking: Folklore VersusFact (accesible en)

- (2000b). H ow to M ake the Right Friends inside Your Company (www.humaxnetworks.com)

- (2000C). Building Social Capital As an Organisational Competence, University of M ichigan Business School W orking Paper.

- (2001). Achieving Success T hrough Social Capital, Ann Arbor, M I, University of M ichigan Business School Press.

Besen, S. M .; FARReLL, J. (1994). «Choosing H ow to Compete: Strategies and Tactics in Standardization», Journal of Economic Perspectives, primavera, 117-131.

Bo LTAN SKI, L.; C H IAPELLO, E. (1999). Le nouvelle esprit du capitalisme, G allimard, París. (trad. castellana El nuevo espíritu del capitalismo, M adrid, Akal, 2002.)

Bo orm AN, S.; BREIGER, R.; W HITE, H.C. (1976). «Social Structure from M ultiple N etworks: I. Blockmodels of Social Positions», American Journal of Sociology, 81, 730-780.

BOURDIEU, P. (2003). Las estructuras sociales de la economía, Barcelona, Anagrama. BoYLE, J. (1996a). Shamans, Software and Spleens. Law and the Information Society, C ambridge, M A, H arvard University Press.

BU RT, R. S. (1988). «T he Stability of American markets», American Journal of Sociology, 94, 365-95.

- (2000). «T he N etwork Structure of Social Capital», en R. I. Sutton y B. Staw (eds.), Research in 0 rganizational Behavior, vol. 22, Greenwich, CT, JAI Press. 
Caballero, P. (1996). Introducción a la criptografía, M adrid, Ra-M a.

COASE, R. (1960). «The Problem of Social C ost», Journal of Law and Economics, 3, 1-44.

D EM SETZ, H . (1986). La competencia. Aspectos económicos, jurídicos y políticos, M adrid, Alianza.

D AVID, P. A. (1986). «Understanding the Economics of QWERTY: the N ecessity of $\mathrm{H}$ istory», en W. N . PARKeR (ed.), Economic H istory and the M odern Economist, O xford, Blackwell, 30-49.

D AVID, P. A.; D Asgu PTA, P. (1994). «Toward a N ew Economics of Science», Research Policy, 23 (5), 487-521.

FERn ÁN DEZ O RDó ÑEZ, M . A. (2000). La competencia, M adrid, Alianza.

Foster, D.; YOUNG, H. P. (1990). «Stochastic Evolutionary Game D ynamics», The oretical Population Biology, 38, 219-232.

Freem AN, L. C. (2000). «La centralidad en las redes sociales. Clarificación conceptual» [1977], Política y Sociedad, 33, enero-mayo, 131-148.

Gran OVetTer, M . S. (1985). «Economic Action and Social Structure: The Problem of Embeddedness», American Journal of Sociology, 91, 481-510.

- (1990). «M odelos de umbral de la acción colectiva» [1978], Zona Abierta, 54/55, 137-166.

- (1992). «T he Sociological and Economic Approaches to Labor M arket Analysis: A Social Structural View», en Gran OVetTer y Swed berg (1992), 233-263.

H EILBRON , J.; C ON VERT, B. (1999). «Reinventing Economic Sociology», ponencia presentada en el IV C ongreso Europeo de Sociología, Amsterdam, 18-21 agosto.

IzQUIERD 0, A. J. (2002a). «D e la fiabilidad. Riesgo financiero y gobierno a distancia», en A. RuBIo (ed.), Finanzas y soci edad, M adrid, Paraninfo, 337-368.

- (2002b). «D elitos, faltas y Premios N obel», Política y Sociedad, 39 (2), 339-359.

- (2002c). «C rítica y consultoría. Para leer El N uevo Espíritu del Capitalismo», Empúria, 5, 145-172.

Katz, M.; ShAPIRO, C. (1994). «Systems Competition and N etwork Effects», Journal of E conomic Perspectives, primavera, 93-115.

- (1998). Antitrust in Software M arkets, University of California at Berkeley, 22 septiembre.

KLEIN , J. I. (1998). The Importance of Antitrust Enforcement in the N ew Economy, conferencia pronunciada en la N ueva York State Bar Association Antitrust Law Section Program, N ew York, 29 de enero.

KLEM PERER, P. (2000). «iSon las subastas lo mejor?», El País, suplemento «N egocios», domingo 19 de noviembre, 10.

Kovacic, W. E.; SH APIRO, C. (2000). «Antitrust Policy: A Century of Economic and Legal Thinking», Journal of Economic Perspectives, primavera, 47-60.

LAN D AUER, R. (1991). «nformation is Physical», Physics Today, mayo, 23-29.

LESSIG, L. (2001). El código y otras leyes del ciberespacio, M adrid, Taurus.

Liebowitz, S. J.; M ARG OLIS, S. E. (1990). «T he Fable of the Keys», Journal of Law and Economics, abril, 1-26.

LYN CH, M.; J ASAN O FF, S. (1998). «C ontested Identities: Science, law and Forensic Practices, Social Studies of Science, 28 (5-6), 675-86.

M AN DELBROT, Benoît (1997). «N ew M ethods in Statistical Economics: Post-Publication Appendix», en M AN D ELBROT, Fractals and Scaling in Finance, Nueva York, primavera, 79-103.

M IROWSKI, P. (2002). M achine D reams. Economics Becomes a Cyborg Science, N ueva York, C ambridge U niversity Press. 
M o LiN A, J. L. (2001). El análisis de redes sociales. U na introducción, Barcelona, Bellaterra.

PADGET, J. F. (1981). «H ierarchy and Ecological Control in Federal Budgetary D ecision- M aking», American Journal of Sociology, 87, 75-129.

PizARRo, N . (1990). Losmétodos de etudio delas organizaciones administrativas, M adrid, Coloquio.

Po d OLN Y, J. M . (1993). «A Status-based M odel of M arket Competition», American Journal of Sociology, 98, 829-872.

PO LAN YI, K. (1976). «La economía como actividad institucionalizada» [1957], en K. Polan yi, C. Aren sberg y H. Pearson (dirs.), Comercio y mercado en losimperios antiguos, Barcelona, Lábor, 289-315.

POSNER, R. (1998). El análisis económico del derecho [1992], M éxico, DF, Fondo de Cultura Económica.

Sala I M ARTín, X. (2002). «El caso M icrosoft», en Sala I M artín, Economía liberal para no economistas y no liberales, Barcelona, Plaza \& Janés, 79-83.

SCHELER, U . (2002). N etworking como factor de éxito. Establezca los contactos adecuados, cuídel os y obtenga beneficios de ello, Barcelona, G estión2000.com.

Sh APIRO, C. (2000). «Competition Policy in the Information Economy», University of California at Berkeley, en Foundations of Competition Policy Analysis, Routledge.

- (1996). Antitrust in N etwork Industries, conferencia pronunciada en el American Law Institute and American Bar Association, Stouffer Stanford Court H otel, San Francisco, 25 de enero.

SWedberg, R.; Gran ovetter, M . (eds.) (1992). The Sociology of Economic Life, Boulder, CO, Westview Press.

VARELA, F.; Th O M PSO N, E.; RosCh , E. (1992). D e cuerpo presente. Las ciencias cognitivas y la experiencia humana, Barcelona, G edisa.

VARIAN, H .; SHAPIRO, C. (2000). El dominio de la información: U na guía estratégica para la economía de la red, Barcelona, Bosch.

W н ITE, H. C. (1973). «Everyday Life in Stochastic N etworks», Sociological Inquiry, $43(1), 43-49$.

- (1980). «W here D o M arkets Come From?», American Journal of Sociology, 87, 517-47.

- (1992). I dentity and Control. A Structural Theory of Social Action, Princeton, N J, Princeton University Press.

Wayner, P. (2001). La ofensiva del software libre, Barcelona, Granica. 